\title{
Hypothyroidism an unseen link between proliferative diabetic retinopathy and type 2 diabetes mellitus
}

\author{
George $\mathrm{E}^{1}$, Kuriakose $\mathrm{S}^{2 *}$, Chackochan $\mathrm{M}^{3}$ \\ 1,2Department OF General MEDICINE, Al Azhar Medical College, Ezhalloor P O, Kerala, INDIA. \\ ${ }^{3}$ Consultant OPHTHALMOLOGIST, Fathima Eye Care Hospital, Thodupuzha, Kerala, INDIA. \\ Email: dredwingeorge@gmail.com, drsuneethkuriakose@gmail.com
}

\section{$\underline{\text { Abstract }}$}

\begin{abstract}
Background: Co existence of hypothyroidism and type 2 diabetes results in accelerated microvascular complications by the dysregulated atherogenesis and deposition of advanced glycosylation products. The aim of the study is to look at the occurrence of retinopathy in diabetic patients with or without thyroid dysfunction Methods: 60 patients with Type 2 diabetes mellitus were enrolled in the study they were divided into two groups of 30 each numbers, a group with hypothyroidism and one with normal thyroid function. Retinopathy occurrence was compared in both groups and possible association was analyzed. Results: Both groups were age and sex matched. The distribution of retinopathy among and patients in the group with thyroid dysfunction and patients in the group without thyroid dysfunction were $42.8 \%$ and $30.4 \%$ respectively, the result was found to be insignificant $(\mathrm{p}$ value 0.559$)$. Proliferative diabetic retinopathy was found to be significantly higher in diabetic patients with thyroid dysfunction ( $p$ value: 0.02 ) Conclusion: There was statistically significant association between proliferative retinopathy in diabetic patients with hypothyroidism. Hence the retinopathy progression is higher in diabetics with hypothyroidism early screening for thyroid dysfunction with normalization of hormone levels is required.
\end{abstract}

Key Words: Hypothyroidism, Diabetes, Retinopathy.

*Address for Correspondence:

Dr Suneeth Kuriakose, Department of General medicine, Al Azhar Medical college, Ezhalloor P O, Kerala, INDIA 685605.

Email: drsuneethkuriakose@gmail.com

Received Date: 04/04/2020 Revised Date: 12/06/2020 Accepted Date: 20/07/2020

DOI: https://doi.org/10.26611/10211525

This work is licensed under a Creative Commons Attribution-NonCommercial 4.0 International License. (oc) EY-NC

\begin{tabular}{|l|l|}
\hline \multicolumn{2}{|c|}{ Access this article online } \\
\hline Quick Response Code: & Website: \\
\hline & www.medpulse.in \\
\hline
\end{tabular}

\section{INTRODUCTION}

Insulin resistance plays a pivotal role in the pathogenesis of type 2 diabetes mellitus(T2DM). Thyroid dysfunction along with reduced GLUT 2 expression, leads to the same ${ }^{1}$. Metabolic syndrome including atherosclerosis, hypertension and cardiovascular disorders has got close relation with thyroid dysfunction. The famous ominous octet of the etiopathogenesis of diabetes mellitus underlines the importance of insulin resistance. Dysregulation of adipocyte metabolism and deposition of advanced glycosylation end products (AGE) resulting in the microvascular complications of diabetes is accelerated by hypothyroidism ${ }^{2}$. Diabetic retinopathy (DR) is one of the major microvascular complications of T2 DM. The major risk factors for developing DR include duration of diabetes, poor glycemic control, dyslipidemia and hypertension. Thyroid hormone axis plays an important role in maintaining the vascular density of retina. Hypothyroidism is associated with preretinal neovascularisation and thyroxine supplementation leads to normalisation of vascular density ${ }^{3}$.

Several mechanisms may be involved in the association between DR and hypothyroidism.

1) Insulin resistance.

Several studies have found that fasting hyperinsulinemia or insulin resistance (IR) was associated with 
hypothyroidism. Previous studies indicated that IR was associated with the presence of DR in T2DM, and the main mechanism was defective fibrinolysis or impaired vasodilation associated with IR. It may be correlated with the reduction of vasodilation ability and fibrosis caused by IR, which led to destruction of retinal vessel and secondary revascularization ${ }^{4}$.

\section{2) Serum $C$ - reactive protein (CRP)}

The level of CRP in patients with subclinical hypothyroidism( $\mathrm{SCH}$ ) was higher than that in non-SCH people. The study of Van Hecke et al.. showed that there was significant relationship between CRP and DR. DR is caused by the inflammation-mediated injury of vascular endothelial cell. Moreover, CRP is recognized as one of the primary and most sensitive acute phase protein in human nonspecific inflammation reaction ${ }^{5}$.

3) The level of serum homocysteine (Hcy)

Homocysteine (Hcy) in SCH patients was much higher than non-SCH people. Hcy is a reactive amino acid about vascular injuries. Looker et al.. demonstrated that elevated levels of Hcy was the risk factor for DR. The reason may be that Hcy could enhance the lipid peroxidation, which leads to increased levels of oxidized low density lipoprotein (OX-LDL), accelerating the progresses of vascular disease. The mRNA expression of VEGF was significantly enhanced with the concentration of Hcy. Higher increase of VEGF was significantly associated with $\mathrm{DR}^{6}$.

\section{4) Oxidative stress}

The activity of paraoxonase 1(PON1) and superoxide dismutase (SOD) in the plasma of $\mathrm{SCH}$ patients is significantly lower than that of normal control. Some studies have shown that oxidative stress was an important risk factor for promoting the occurrence and development of $\mathrm{DR}^{7}$.

\section{5) Dyslipidemia}

Atherogenic disturbances in lipid metabolism have been observed in patients with hypothyroidism. Thus, dyslipidemia in SCH may be one of the reasons for the association between DR and hypothyroidism ${ }^{8}$.

\section{MATERIALS AND METHODS}

This study is a cross-sectional study done over a period of 18 months. The study was conducted in Victoria hospital affiliated to Bangalore medical college and research institute. Duration of the study was Eighteen months from September 2013 to February 2015.60 patients were enrolled in the study diagnosed with T2 DM, who were willing to participate in the study were evaluated and compared as per the proforma designed for the purpose of the study. As per the aim of the study, the comparison was done among two groups with 30 patients in each group. Thyroid function tests was done in all the patients and 2 groups was made, the first group included diabetic patients with thyroid dysfunction and the second group included diabetic patients without thyroid dysfunction. Fundoscopy was done in all patients by the ophthalmologist for assessing retinopathy. An inclusion criterion was T2 DM patients aged more than 18 years. Exclusion criteria were patients taking drugs affecting thyroid profile and patients with known hypertension, ischemic heart disease, smokers and chronic alcoholics.

Statistical analysis

Statistical analysis was done using spss 16 software. Since the distribution of data was parametric, Chi square test and Fischer's exact test were used to study the differences between categorical data. Student ' $t$ ' test was used to assess associations between numerical variables.

\section{RESULTS}

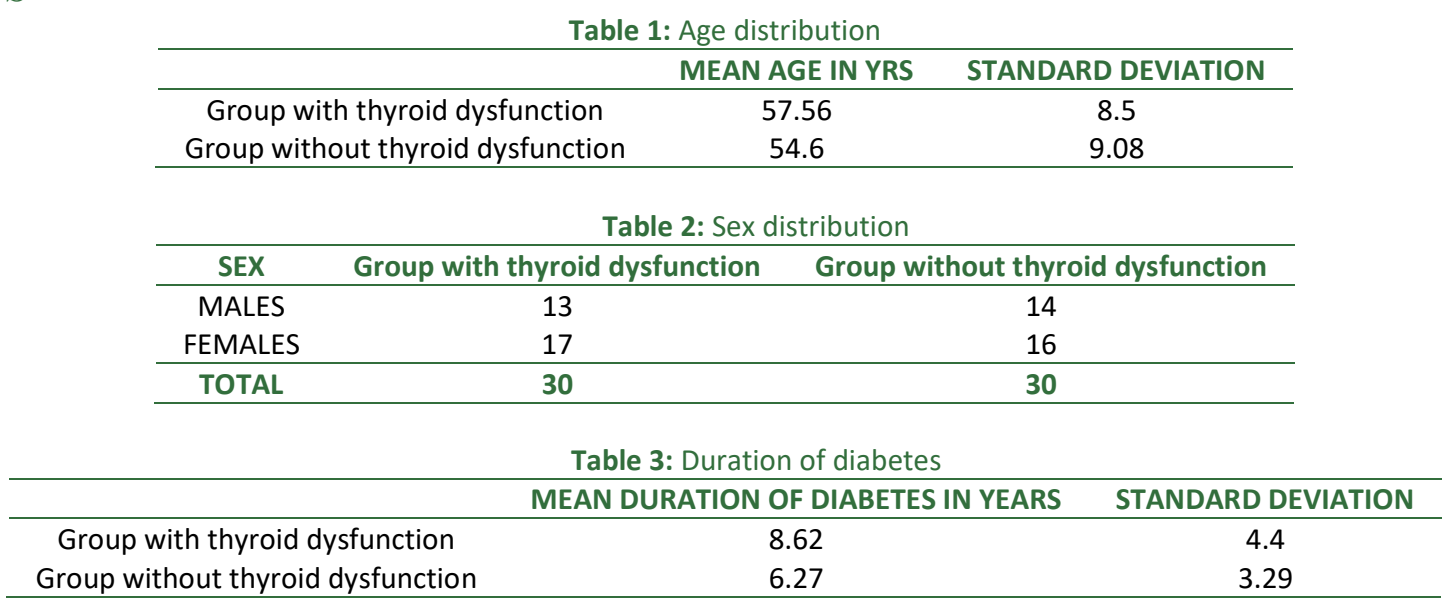


Table 4: Occurrence of retinopathy

\begin{tabular}{ccc}
\hline RETINOPATHY & Group with thyroid dysfunction & Group without thyroid dysfunction \\
\hline PRESENT & 9 & 7 \\
ABSENT & 21 & 23 \\
\hline \multicolumn{3}{c}{ Table 5: Occurrence of proliferative retinopathy } \\
\hline PDR & Group with thyroid dysfunction & Group without thyroid dysfunction \\
\hline PRESENT & 5 & 0 \\
ABSENT & 25 & 30 \\
\hline
\end{tabular}

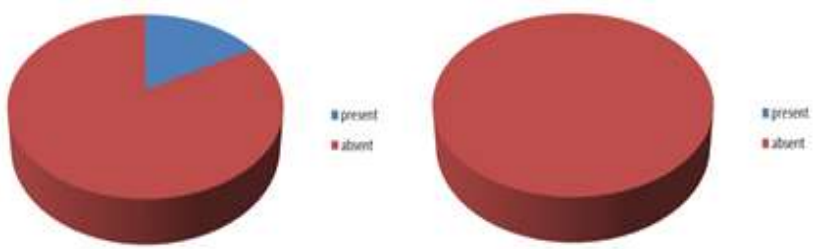

Figure 1: Occurrence of proliferative retinopathy

Sixty patients with T2 DM were divided into two groups of 30 each- those with thyroid dysfunction and those without thyroid dysfunction. These patients were the outpatients or inpatients of Victoria hospital affiliated to Bangalore medical college and research institute. The study was conducted over a period of 18 months.

Age distribution

The minimum age of diabetic patients in the group with thyroid dysfunction was 41 years and the maximum age was 75 years; with a mean age of 57.56 years and standard deviation of 8.5. Minimum age of diabetic patients in the group without thyroid dysfunction was 35 years and the maximum age was 68 years; with a mean age of 54.6 years and standard deviation of 9.08 .

\section{Patient characteristics}

\section{Age distribution}

The minimum age of patients in the group with thyroid dysfunction was 41 years and the maximum age was 75 years; with a mean age of 57.56 years and standard deviation of 8.5. Minimum age of patients in the group without thyroid dysfunction was 35years and the maximum age was 68years with a mean age of 54.6years and standard deviation of 9.08yrs. The mean age of cases and control was matched. (Table 1)

\section{Sex distribution}

The male and female distribution among patients in the group with thyroid dysfunction was $43.3 \%$ and $56.7 \%$ respectively, and that of patients in the group without thyroid dysfunction was $46.66 \%$ and $53.44 \%$ respectively. The gender distribution was matched. (Table 2)

\section{Duration of diabetes}

Among patients in the group with thyroid dysfunction, the duration of diabetes was minimum 1 year, maximum 20 years with a mean duration of 8.62 years and standard deviation of 4.4 years. The duration of diabetes was minimum 6 months, maximum 13 years with a mean of
6.27 years and standard deviation of 3.29 years among patients in the group without thyroid dysfunction. (Table 3)

Retinopathy

The distribution of retinopathy among and patients in the group with thyroid dysfunction and patients in the group without thyroid dysfunction were $42.8 \%$ and $30.4 \%$ respectively, the result was found to be insignificant ( $p$ value 0.559$)($ Table 4$)$

Proliferative diabetic retinopathy

Proliferative diabetic retinopathy was found to be significantly higher in diabetic patients with thyroid dysfunction ( $p$ value: 0.02 ) (Table5) (Figure 1)

\section{DISCUSSION}

A descriptive study was done among 60 patients with known type 2 diabetes mellitus who attended the Medicine OPD or were admitted in the medical wards of Victoria Hospital. The patients were divided into two groups; based on the presence or absence of thyroid dysfunction, with 30 patients in each group, and a comparison between the groups was done with respect to the retinopathy. Baseline thyroid function tests - TSH/T3/T4 was done in all patients and those with thyroid dysfunction were included in one group and patients without thyroid dysfunction were included in the other group. In this study, all the patients in the group with thyroid dysfunction were found to be hypothyroid. The age of patients in the group with thyroid dysfunction ranged from 41-75 years, mean age 57.56 \pm $8.553 \mathrm{yrs}$. The age of patients in the group without thyroid dysfunction ranged from 35-68 years, mean age 54.6 \pm 9.08 yrs. The male and female distribution among the former group was $43.3 \%$ and $56.7 \%$ respectively, and that of latter was $46.66 \%$ and $53.44 \%$ respectively. The difference in mean age and sex was not statistically significant ( $p$ value of 0.162 and 0.795 respectively). In the 
group with thyroid dysfunction, the duration of diabetes ranged from $1-20$ years with a mean duration of 8.62 years and standard deviation of 4.4 years. In the group without thyroid dysfunction duration of diabetes ranged from 6 months- 13 years with a mean of 6.27 years and standard deviation of 3.29 years. The duration of diabetes between the two groups was not statistically significant ( $p$ value 0.328 ).

\section{Retinopathy}

The distribution of retinopathy among the groups with thyroid dysfunction and without thyroid dysfunction was $42.8 \%$ and $30.4 \%$ respectively. This result was found to be insignificant ( $\mathrm{p}$ value 0.559 ). Non proliferative diabetic (NPDR) retinopathy accounted for $57 \%$ of diabetic retinopathy (DR) cases in patients in the group with thyroid dysfunction. Only non proliferative diabetic retinopathy was present in the patients with DR in the group without thyroid dysfunction. The relation was found to be insignificant ( $\mathrm{p}$ value 0.317 ). The study by Chen et al. also showed that thyroid dysfunction did not account for the occurrence of overall retinopathy. Many studies found that the severity of sight threatening retinopathy was more among hypothyroid patients ${ }^{9}$. Kim et al. in their similar study found association between retinopathy and thyroid dysfunction. However the duration of DM in their study was significantly different with more duration in patients with thyroid dysfunction ${ }^{10}$. Proliferative diabetic retinopathy was found to be significantly higher in diabetic patients with thyroid dysfunction ( $p$ value: 0.02 ). This was similar to the studies done by Yang et al. in Beijing and Dan et al. in Shenyang ${ }^{11,12}$.

\section{CONCLUSION}

Diabetic retinopathy overall was not showing any statistically significant association with hypothyroidism however there was statistically significant association between proliferative retinopathy in diabetic patients with hypothyroidism. Hence the retinopathy progression is higher in diabetics with hypothyroidism early screening for thyroid dysfunction with normalization of hormone levels is required.

\section{REFERENCES}

1. Perros P, McCrimmon R J, Shaw G, Frier B M. Frequency of thyroid dysfunction in diabetic patients: value of annual screening. Diabetic Medicine.1995; 12: 622-7.

2. Wang C. The Relationship between Type 2 Diabetes Mellitus and Related Thyroid

Diseases. Journal of Diabetes Research. 2013; 2013:1-9.

3. Mookadam M, Lezke DA, Fautsch MP, et al... The antithyroid drug methimazole induces neovascularisation in neonatal rat similar to ROP.2004; 45: 4145-4150

4. Tuzcu, A, Bahceci M, Gokalp D, et al... Subclinical hypothyroidism may be associated with elevated high sensitive CRP and fasting hyperinsulinemia. Endocrinology Journal. 2005; 52: 89-94.

5. VanHecke M. V. Inflammation and endothelial dysfunction are associated with retinopathy: The Hoom Study. Diabetologia. 2005; 48: 1300-06.

6. Looker H C.Homocysteine as a risk factor for nephropathy and retinopathy in Type 2 diabetes. Diabetologia. 2003; 46:766-72.

7. Baskol G. The role of advanced oxidation protein products and total thiols in diabetic retinopathy. European Journal of Ophthalmology. 2008; 18:792-820.

8. Althaus B U. LDL/HDL-changes in subclinical hypothyroidism: possible risk factors for coronary heart disease. Clinical Endocrinology Oxford. 1988; 28:157-63.

9. Chen $\mathrm{H} \mathrm{S}, \mathrm{Wu} \mathrm{E} \mathrm{J}$ W, Jap $\mathrm{T}$ E, et al... Subclinical hypothyroidism is a risk factor for nephropathy and cardiovascular diseases in Type 2 diabetic patients. Diabetic Medicine. 2007; 24: 1336-44.

10. Kim B Y, Kim C H, Jung C H, et al... Association between subclinical hypothyroidism and severe diabetic retinopathy in Korean patients with type 2 diabetes. Endocrine Journal. 2011; 58:1065-70.

11. Yang GR, Yang J K, Zhang L, et al... Association between subclinical hypothyroidism and proliferative diabetic retinopathy in type 2 diabetic patients: A case control study. Tohoku Journal of Experimental Medicine. 2010; 202: 303310 .

12. Dan G, Bo W. Correlation Analysis of Subclinical Hypothyroidism and Microvascular Complications in Type 2 Diabetic Patients. Journal of China Medical University. 2015; 44: $730-733$

\begin{tabular}{l} 
Source of Support: None Declared \\
Conflict of Interest: None Declared \\
\hline
\end{tabular}

Policy for Articles with Open Access:

Authors who publish with MedPulse International Journal of Medicine, Print ISSN: 2550-7583, Online ISSN: 2636-4751 agree to the following terms: Authors retain copyright and grant the journal right of first publication with the work simultaneously licensed under a Creative Commons Attribution License that allows others to share the work with an acknowledgement of the work's authorship and initial publication in this journal.

Authors are permitted and encouraged to post links to their work online (e.g., in institutional repositories or on their website) prior to and during the submission process, as it can lead to productive exchanges, as well as earlier and greater citation of published work. 